\title{
Stéphanie KUNERT, Publicité, Genre et Stéréotypes
}

Fontenay-le-Comte, Lussaud, coll. L'Impensé contemporain, 2013, 197 pages

\section{Stéphane Héas}

\section{CpenEdition}

\section{Journals}

Édition électronique

URL : http://journals.openedition.org/questionsdecommunication/9909

DOI : 10.4000/questionsdecommunication.9909

ISSN : 2259-8901

\section{Éditeur}

Presses universitaires de Lorraine

\section{Édition imprimée}

Date de publication : 1 septembre 2015

Pagination : 378-379

ISBN : 9782814302600

ISSN : 1633-5961

\section{Référence électronique}

Stéphane Héas, "Stéphanie kunert, Publicité, Genre et Stéréotypes 》, Questions de communication [En ligne], 27 | 2015, mis en ligne le 01 septembre 2015, consulté le 23 septembre 2020. URL : http:// journals.openedition.org/questionsdecommunication/9909; DOI : https://doi.org/10.4000/ questionsdecommunication.9909 
de la rigueur théorique du travail universitaire qui le précède. Cependant, ce qui pourrait être perçu un peu rapidement comme une réflexion aux accents scolaires est vite compris comme étant une nécessité pour appréhender ce milieu caractérisé par son hétérogénéité Oscillant entre sociologie, histoire, économie et politique, et par un brillant mélange d'analyses et de témoignages l'ouvrage trouve très justement sa place dans la collection «Socio-Histoires » dirigée par Michel Offerlé. Les nombreux extraits d'entretiens qui structurent le texte permettent de saisir les questions qui gravitent autour des métiers d'art de façon presque sensible. L'ouvrage offre une lecture très souple tout en livrant une pensée minutieusement organisée pour tenter d'apporter un semblant de réponse à « l'insaisissable définition des métiers d'art » pour reprendre les mots de Pierre Dehaye en 1976 lors du rapport fondateur de l'Institut national des métiers d'Art (p. 12). Mais la finalité n'est pas tant de trouver des réponses que de révéler les questionnements soulevés par cette population dont on parle peu et quand bien même on trouve des écrits à leur propos, c'est souvent avec les stéréotypes évoqués par les artisans lors des entretiens avec l'auteure. Anne Jourdain tente de traduire cette diversité des métiers, des pratiques, des styles de production, des circuits de commercialisation pour saisir un tant soit peu cette catégorie polysémique forte d'une construction à la fois collective et individuelle. Construction collective autour d'un marché, celui de l'artisanat d'art, mais aussi une construction individuelle visible par la diversité des parcours. Ce groupe n'a, historiquement, pas toujours été distingué de l'art. La conséquence du clivage entre art et artisanat serait une absence de définition substantive de ce premier groupe ainsi qu'une absence de statut qui soit spécifique aux artisans d'art. Anne Jourdain relève scrupuleusement les différentes tentatives de définition des organismes concernés avant de rappeler que, s'il existe un groupe « artisanat d'art », il est le fruit d'une construction sociale et historique impliquant les effets de multiples acteurs qui ont intérêt à l'institutionnalisation de ce groupe. Et c'est de cette construction dont il est précisément question ici.

Le régionalisme de la fin du XIX $x^{e}$ siècle, qui trouve son apogée dans les années 30 à travers la valorisation du folklore, contribue par exemple à la promotion des métiers d'art régionaux en France. L'apparition de la figure de l'artiste indépendant ou encore la loi sur la durée du service militaire (loi qui vise à dispenser du service militaire de trois ans un certain nombre d'ouvriers d'art et doit donc définir au préalable ce qu'elle entend par métiers d'art ou industrie d'art) auront également une incidence (p. 24). Progressivement, ce sont les artistes qui vont encourager le développement de ces métiers en s'intéressant, dès 1930, aux savoir-faire artisanaux. Les écoles d'art suivront en renouant avec une certaine idéologie artisanale, encourageant ainsi une réhabilitation artistique de l'artisanat. Dans les années 70, les premiers métiers d'art, issus de la Révolution industrielle, se chargent des revendications esthétiques et éthiques contre la civilisation urbaine et industrielle. Ce retour à la nature encourage d'avantage ce qui sera désormais qualifié d'artisanat d'art ou d'artisanat créateur (p. 29).

Le sens fluctue autour de ce terme qui paraît pourtant clair, les politiques viennent le modeler sans jamais le figer. Les systèmes de pensée s'y heurtent et dépassent le vieux débat entre l'artiste et l'artisan, certains voient dans les métiers d'art une possibilité d'extension de l'art contemporain.

Les possibles sont multiples et c'est à coup d'étiquetage et de changement de ces étiquettes que les frontières, poreuses, faute de se dessiner, semblent s'élargir voire s'ouvrent aux hybridations. L'apparente incompatibilité entre tradition et création, la réappropriation des images de l'artisanat d'art par le luxe, les difficultés économiques qui touchent les artisans sont autant de problématiques rencontrées lors de la lecture de cet ouvrage dans lequel l'auteure a mis, indubitablement, beaucoup de cœur.

\section{Tiphaine Marie \\ Crem, université de Lorraine, F-57000 tiphaine.mariel6@gmail.com}

Stéphanie Kunert, Publicité, Genre et Stéréotypes.

Fontenay-le-Comte, Lussaud, coll. L'Impensé contemporain, 2013, 197 pages

L'objectif de cet ouvrage au format non standard (en « paysage ») est de mieux saisir «l'économie discursive des messages publicitaires » ayant trait aux minorités sexuelles et plus largement aux stéréotypes de genre (p. 23). Le livre est divisé en six parties d'environ 12 à 16 pages sauf l'une d'entre elle, « Les fonctions d'illustration de I'homosexualité en publicité » (pp.67-129), qui comporte 62 pages; les sous-chapitres ne sont pas indiqués dans le sommaire. Résultat :l'ensemble peut paraitre déséquilibré. L'auteure, docteure en sciences de l'information et de la communication, analyse la construction-exposition des orientations sexuelles et du genre dans les médias... ce qui aurait pu faire l'objet d'un judicieux sous-titre à l'ouvrage.

La préface écrite par Karine Berthelot-Guiet rappelle que la publicité a toujours comme objectif « de convaincre pour vendre » (p. 12). lci, cette « médiation marchande » est décryptée en tant que « technologie du genre 
stéréotypique ». Les analyses déployées soulignent « le mouvement paradoxal que Stéphanie Kunert nomme « dé-figement/réification 》 des stéréotypes liés aux genres (p. 13). Une « attitude réflexive » relative à la perspective sémiologique de l'image publicitaire est déployée à la suite des travaux princeps de Roland Barthes (Mythologies, Paris, Éd. Le Seuil, 1957 ; « Rhétorique de l'image », Communications, 4, 1964, pp. 40-5I), dans le prolongement de ceux de Karine Berthelot-Guiet (« Instrumentalisation de la sémiotique », Études de communication, 27, 2004, accès : http://edc. revues.org//48) justement, ou bien ceux de Jean-Jacques Boutaud et Eliseo Veron (Sémiotique ouverte. Itinéraires sémiotiques en communication, Paris, Lavoisier, 2007).

La méthodologie mobilisée est concentrée plus spécifiquement sur 120 annonces diffusées dans les médias de masse et 80 parues dans les médias spécifiques aux « minorités sexuelles et de genre » (p. 20), expression préférée à la dénomination LGBT (lesbiennes, gays, bi et trans) ou LGBTI (lesbiennes, gays, bi, trans et intersexuels) considérée largement comme anachronique. De cette population d'étude, ont cependant été exclues les annonces et campagnes de publicité endogènes, c'està-dire émanant directement des organisations LGBTI. Quelques rares éléments du codage sont précisés rapidement comme les fonctions d'illustration avec la présence des personnes LBGTI dans les publicités versus la fonction d'adresse lorsque les minorités sont destinataires des messages/annonces publicitaires (p. 67). La fonction comique des publicités recourant aux minorités sexuelles et de genre est plus particulièrement analysée.

À partir des propositions de la sociologie des tribus de Michel Maffesoli, enrichies de la démarche communicationnelle de Bernard Cathelat notamment, un gay marketing a pu être élaboré progressivement ces dernières décennies. Ce ciblage médiatico-commercial a eu comme répercussion principale la naissance d'un gay marketing et de la figure largement homogénéisante du gay consommateur dans les années 2000 en France. Toutefois, l'eldorado annoncé de ce nouveau marché prometteur n'a, semble-t-il, pas perduré malgré une organisation spécifique tel le Syndicat national des entreprises gaies (SNEG) depuis 1990. Surtout, la focalisation induite par le terme même gay marketing réduit considérablement les réalités des minorités sexuelles et genrées, confirmant la masculinisation de I'homosexualité, elle-même noyée dans la masculinité hégémonique et hétérosexuelle des messages publicitaires. Pour l'auteur, ce ciblage et cette apparition aux intérêts économiques bien compris n'ont pas permis de réduire les stéréotypes liés aux minorités sexuelles et de genre, au contraire. En effet, suivant Montserrat
Lopez-Diaz, le « détournement, la déstructuration, la transgression » des normes dans les messages publicitaires a renforcé la norme selon le principe que « le défigement réifie le figement, la transgression réifie la norme » (p.69). La fonction comique mobilisée lorsque des personnes aux orientations sexuelles ou genrées minoritaires sont présentes dans les publicités confirme au final cette trivialisation, et par conséquent cette valorisation plus ou moins explicite de la norme hétérosexuelle en même temps que la polarisation genrée. Tout se passe comme si les publicités, en cherchant à séduire de nouvelles niches, confirmaient un processus d'altérisation de ces minorités

L'auteure en appelle au développement d'une « sémioclastie militante » visant à casser les stéréotypes comme autant de « cage(s) sémantique(s) dont i faut tordre les barreaux » (p. 155). La dénomination « minorités sexuelles et genrées » elle-même s'avère contre-productive puisqu'elle souligne, elle aussi, la référence à la doxa, à la norme, à la majorité dominante. En définitive, l'auteure rappelle que la publicité est normative dans la mesure où elle ne reflète pas tant la réalité que la réalité telle qu'elle est envisagée par celles et ceux qui font profession de publicitaire ou bien qui ont recours à leurs services. En ce sens, tel un « miroir normatif » (p. 174), la publicité ressemble à s'y méprendre à un centrisme professionnel, fermé à la richesse même de la réalité socioculturelle d'un pays à un moment donné.

Ce petit ouvrage offre notamment dans le chapitre le plus important des illustrations de cette doxa publicitaire qui altérise les Autres sous l'angle des sexes et des genres. En refermant le livre, l'impression reste que les concepts intéressants sont présentés très tardivement telle la distinction proposée par Stéphane Legrand entre « normation », normativité et normalisation ou bien le projet ambitieux de sémioclastie. Cette analyse a toutefois le mérite de préciser les processus insidieux de dégradation sociale et culturelle orchestrés par les annonces publicitaires. Elle permet également de constater ce phénomène à partir d'exemples publicitaires précis, tout en indiquant des sites internétiques par exemple où trouver des collections de publicités (audio et vidéo notamment). C'est le cas par exemple de l'activité de l'Institut national de l'audiovisuel bien sûr, mais aussi de l'association Commercial Closet, etc. Ces analyses constituent surtout une étape intéressante pour aller plus loin dans l'approfondissement de ces discriminations qui ne disent pas leur nom...

Stéphane Héas

VIP\&S, université de Rennes, F-35043 stephane.heas@univ-rennes2.fr 